\title{
CARTOX Grade 3
}

National Cancer Institute

\section{Source}

National Cancer Institute. CART OX Grade 3. NCI Thesaurus. Code C155298.

A score of 0-2 on the CART OX-10 assessment, indicating severe neurotoxicity. 